Claremont Colleges

Scholarship@ Claremont

WM Keck Science Faculty Papers

W.M. Keck Science Department

$10-1-1996$

\title{
Dynamical Effects of Partial Orderings in Physical Systems
}

\author{
Adam S. Landsberg \\ Claremont McKenna College; Pitzer College; Scripps College
}

Eric J. Friedman

Cornell University

\section{Recommended Citation}

Landsberg, A.S., and E.J. Friedman. "Dynamical Effects of Partial Orderings in Physical Systems." Physical Review E 54.4 (1996): 3135-3141. DOI:10.1103/PhysRevE.54.3135

This Article is brought to you for free and open access by the W.M. Keck Science Department at Scholarship @ Claremont. It has been accepted for inclusion in WM Keck Science Faculty Papers by an authorized administrator of Scholarship @ Claremont. For more information, please contact scholarship@cuc.claremont.edu. 


\title{
Dynamical effects of partial orderings in physical systems
}

\author{
A. S. Landsberg* \\ Physics Department, Georgia Institute of Technology, Atlanta, Georgia 30332 \\ Eric J. Friedman ${ }^{\dagger}$ \\ Fuqua School of Business, Duke University, Durham, North Carolina 27708-0120
}

(Received 22 November 1995)

\begin{abstract}
We demonstrate that many physical systems possess an often overlooked property known as a partialordering structure. The detection and analysis of this special geometric property can be crucial for understanding a system's dynamical behavior. We review here the fundamental dynamical features common to all such systems, and describe how the partial ordering imposes interesting restrictions on their possible behavior. We show, for instance, that though such systems are capable of displaying highly complex and even chaotic behaviors, most of their experimentally observable behaviors will be simple. Partial orderings are illustrated with examples drawn from many branches of physics, including solid state physics, fluids, and chemical systems. We also describe the consequences of partial orderings on some simple nonlinear models, and prove, for example, that for general two-dimensional mappings with the partial-ordering property, period 3 implies chaos, in analogy with the well-known result of Li and York [Am. Math. Mon. 82, 985 (1975)] for (ordinary) one-dimensional mappings. [S1063-651X(96)01308-6]
\end{abstract}

PACS number(s): 05.45.+b, 02.30.Hq, 03.20.+i, 03.40.-t

\section{INTRODUCTION}

Physical systems often possess some special underlying structure or property, which, once recognized, can prove vital for understanding their dynamical behavior. The two most common and easily identifiable of these are Hamiltonian structures and spatial symmetries, which arise in numerous problems of physical interest. As is well known, each can have a dramatic influence on the types of behaviors seen in a system; the former, for example, immediately precludes the possibility of strange attractors owing to the conservation of phase space volumes, while the latter commonly gives rise to conservation laws and multiple bifurcations. This paper is devoted to understanding a third type of special structure which frequently emerges in physical systems, called a partial ordering. This special geometric property, though not as common as the other two, is nonetheless present in a remarkable number of problems in many diverse areas of physics, yet it often goes unrecognized, and hence has received surprisingly little attention within the physics community. When it has been recognized, it has been studied largely on a case by case basis. We seek here to analyze such systems within a single unifying framework, and to illustrate explicitly the qualitative dynamical features that will be common to all physical systems with this property.

Partial-ordering structures have previously garnered attention in the mathematics, biology, and economics communities, where they arise, for example, in studies of isotone mappings of complete lattices (e.g., [1-5]). Accordingly, a great deal is already known about such systems (see [6-8], in

\footnotetext{
*Present address: Department of Physics, Haverford College, Haverford, PA 19041.

${ }^{\dagger}$ Present address: Department of Economics, Rutgers University, New Brunswick, NJ 08903.
}

particular, and [9] for a recent review). Our primary aim here is to demonstrate the relevance of partial orderings to systems of direct interest to the physics community, and to show how this structure can provide the key to understanding their dynamical behavior. By design, this paper represents an amalgam, combining a review of many known theoretical results (with simplified proofs), a presentation of additional results, and explicit illustrations based on physical examples. We will describe how the partial-ordering constraint imposes significant restrictions on the dynamics of a system, yet still allows for interesting behaviors (e.g., chaotic sets, period doublings, global bifurcations). For one-dimensional systems, the partial ordering will reduce to a total ordering, and the dynamics will prove to be trivial (though still potentially useful [10-12]). However in higher dimensions the situation is much more complicated, and arbitrarily complex (i.e., chaotic) behavior can arise. However, unlike what occurs in other systems, the partial-ordering structure will force all chaotic motion to be unstable, so that the long-term, physically observable behaviors in partially ordered systems will not be chaotic for most initial conditions. We also describe the geometric implications of partial-ordering structures on some simple, slightly more abstract nonlinear systems as well. As an example, we will show that the famous Li-Yorke theorem [13] for ordinary one-dimensional maps ("periodthree implies chaos"') also holds for two-dimensional maps with a partial-ordering structure.

This paper is organized as follows. In Sec. II we define what is meant by a partial ordering, and present a simple criterion by which it may be recognized in a physical system. Section III provides examples of partially ordered systems taken from different areas of physics, and Sec. IV describes their basic dynamical properties.

\section{THE BASICS}

In its simplest terms, a physical system is partially ordered if, whenever one solution is "larger' (in some appro- 


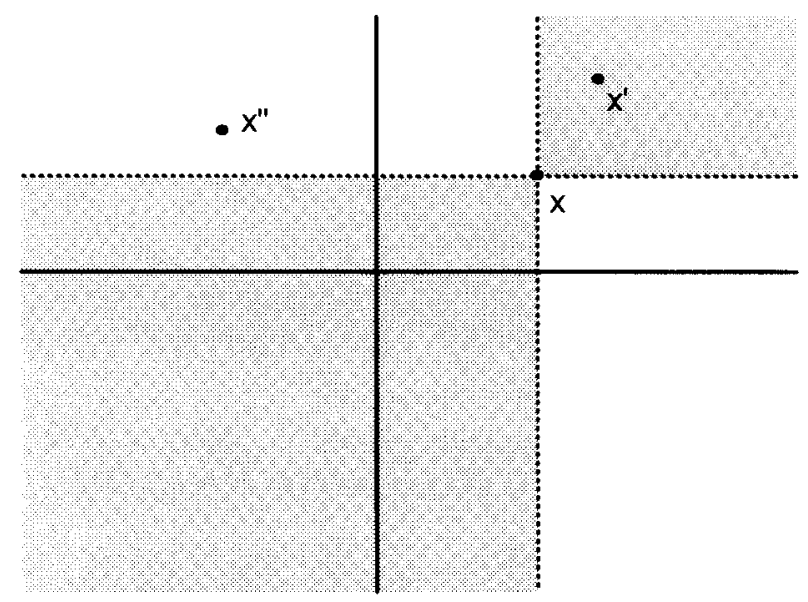

FIG. 1. The shaded regions show the points in the plane which are ordered with respect to the point $x$. Those in the upper region (e.g., $x^{\prime}$ ) are larger than $x$, while those in the lower region are smaller. The points $x^{\prime \prime}$ and $x$ are incomparable. Note that points which are ordered with respect to $x$ need not be ordered with respect to each other.

priate sense) than another, it remains larger for all time. We might consider, for instance, two waves in a fluid, and define one wave to be larger than a second if the amplitude of the first wave at each point in space exceeds that of the second at the corresponding point. If this ranking is preserved under the flow, the fluid system is said to have a partial-ordering structure. It is an "ordering" because various behaviors in the system can be ranked according to size; it is "partial", because not all solutions can be directly compared (e.g., one wave might be larger than a second in one region of the container, but smaller in another).

We can formalize this notion as follows. The dynamical behavior of a general system can be described in terms of its time evolution operator $\hat{\phi}_{\tau}$, taking a point $x \in \mathbb{R}^{n}$ in phase space to a new point $\hat{\phi}_{\tau}(x)$ at time $\tau$ later, $\hat{\phi}_{0}$ being the identity operator. For simplicity we assume that phase space is finite dimensional, but allow time $\tau$ to be either continuous (e.g., ordinary differential equations) or discrete $\tau \in(0,1,2, \ldots)$ (mappings). (The generalization to continuum models described by partial differential equations is straightforward.) Next define $\leq$ to be a partial-ordering relation on the phase space of the system; the canonical example is the ordering relation defined by: $x \leq x^{\prime}$ if $x_{i} \leqslant x_{i}^{\prime}$ for all components $i=1, \ldots, n$. This simple ordering relation provides a potential way of comparing two points in phase space: one point (i.e., vector) $x^{\prime}$ is considered larger than a second point $x$ if each component of the first equals or exceeds the corresponding component of the second (Fig. 1). Two points that can be compared in this manner are called ordered or comparable. It is crucial to recognize that $\leq$ defines a partial ordering, and hence not all points in phase space can be ordered [e.g., in two dimensions consider points $(2,0)$ and $(3,-1)]$; such points are said to be unordered or incomparable. Note that the ordering relation is transitive, so that if comparable points $x, x^{\prime}$ satisfy $x \leq x^{\prime}$, and $x^{\prime} \leq x^{\prime \prime}$, then $x \leq x^{\prime \prime}$. Note too that if $x \leq x^{\prime}$ and $x^{\prime} \leq x$, then $x$ and $x^{\prime}$ are necessarily the same point. We now define a partially ordered dynamical system as follows.
Partial ordering: A dynamical system has a partialordering structure if, for any $x, x^{\prime} \in \mathbb{R}^{n}$ satisfying $x \leq x^{\prime}$, it follows that $\hat{\phi}_{\tau}(x) \leq \hat{\phi}_{\tau}\left(x^{\prime}\right)$ for all times $\tau \geqslant 0$.

This definition requires that the partial ordering on the phase space be preserved under time evolution, guaranteeing that if one state is initially "larger' than a second state, then it remains larger under the dynamics.

Before considering some examples of physical systems with a partial ordering, we first describe a simple criterion to more easily test for the presence of this special structure. Specifically, we show that the (global) partial-ordering property defined above implies that a certain local ordering constraint must also be satisfied, and further, that this local ordering constraint is in fact sufficient to generate the global partial-ordering structure. Certain distinctions exist between the cases of discrete and continuous flows, and we note these accordingly. In particular, we have the following.

Local ordering criterion [14]: For any partially ordered, differentiable map $x \rightarrow f(x)$, all elements of the Jacobian matrix $D f(x)$ are non-negative for all $x$. For any partiallyordered vector field flow $d x / d t=f(x)$, all off-diagonal elements of $D f(x)$ are non-negative for all $x$.

The proof of these statements follows by considering any two arbitrarily close, ordered points $x, x+\Delta x$. For the sake of argument assume $0 \leq \Delta x$. Under one iteration of the mapping $f$, the separation between the two points becomes $D f(x) \Delta x$. Since the original points were ordered, by definition they must remain ordered, so that $0 \leq D f(x) \Delta x$. Since this must hold for any $\Delta x$ satisfying $0 \leq \Delta x$, each component of the matrix $D f(x)$ must be non-negative. The corresponding condition for continuous flows is proven similarly.

The above constraint on the Jacobian represents a local criterion, guaranteeing that if two neighboring points are ordered, they remain ordered under the dynamics provided they remain close. To show that this local constraint is sufficient to generate the full global structure, we note that any two ordered points (not necessarily close) can be connected by a straight line, with all points on the line being ordered (i.e., comparable). As this line evolves under the dynamics, the non-negativity constraint on the Jacobian guarantees that the ordering will be preserved locally for all points on this line. Hence, since the ordering relation is transitive, the two end points of the line will remain ordered.

\section{PHYSICAL SYSTEMS WITH PARTIAL ORDER}

Partial orderings appear in physical systems in a variety of settings. We now illustrate this with several examples taken from different areas of physics, and later describe how this special structure affects their dynamical behavior.

(1) Charge-density waves: Middleton and Fisher $[15,16]$ studied a model for sliding charge-density waves (CDW's), and were able to predict some striking results concerning the sliding behavior of these states. These results were based on a peculiar property of the system which they dubbed a "nopassing rule,' indicating that CDW's cannot pass one another. The model equations are written in terms of variables $\phi_{i}, i=1, \ldots, N$ describing the distortion of the CDW at the $N$ lattice sites, and take the form 


$$
\frac{d \phi_{i}}{d t}=h \sin \left(\phi_{i}-\beta_{i}\right)+F+\sum_{\{j\}}\left(\phi_{i+j}-\phi_{i}\right), \quad i=1, \ldots, N,
$$

where the first term represents a pinning force, the second $(F)$ a driving field, and the third the elastic forces between neighboring lattice sites. A quick inspection reveals this system always has non-negative off-diagonal Jacobian matrix elements (owing to the nature of the elastic forces), and so by the local ordering criterion described above, we can immediately conclude that this system has the partial-ordering property. The no-passing rule is directly related to this special structure-the elastic forces prevent CDW's from passing one another, thereby setting up a partial ordering. We observe too that Eqs. (1) also have a discrete symmetry property: if all variables $\phi_{i}$ are simultaneously increased by $2 \pi$, Eqs. (1) remain unchanged (i.e., equivariant). Using this fact in conjunction with the no-passing rule, Middleton and Fisher were able to obtain even stronger results for their system, showing in fact that all sliding CDW's will move with the same average velocity. We will return to this point later, and show that it is a general feature of partially ordered systems with certain discrete symmetries.

(2) Mode interaction in fluids: Near an instability, the behavior of a fluid can often be described in terms of a finite number of spatial modes. One rather well-known example is when three modes of the system simultaneously become unstable, as might arise in various fluid convection problems $[17,18]$. Under appropriate conditions, this process is described by equations of the form

$$
\begin{aligned}
& \frac{d z_{1}}{d t}=z_{2}, \\
& \frac{d z_{2}}{d t}=z_{3}, \\
& \frac{d z_{3}}{d t}=-\lambda z_{1}-\nu z_{2}-\eta z_{3}+a z_{1}^{3},
\end{aligned}
$$

where $z_{1}, z_{2}, z_{3}$ denote the three modal amplitudes, and coefficients $\lambda, \quad \nu, \quad \eta, a$ are control parameters of the system. For particular values of the control parameters, these equations are known to exhibit global bifurcations leading to strange attractors $[17,18]$. If we now consider the local ordering criterion, we see that the Jacobian will have nonnegative off-diagonal elements provided $a>0, \lambda<0, \nu<0$. Hence, for this range of parameters, system (2) possesses a partial-ordering structure. As will be shown in the next section, the presence of a partial ordering will immediately preclude the existence of attracting, chaotic orbits. Thus, with minimal effort, we have identified a range of parameters for which there can be no strange attractors anywhere in the system. (The partial-ordering structure will have other dynamical consequences as well, to be described later.)

The above example can be used to illustrate another important point: the partial-ordering relation $(\leq)$ used here is not preserved under coordinate transformations. In some sense, this is a shortcoming, since coordinate-free definitions are often preferable. In practice though, this is seldom a problem and can even be exploited, since a system which

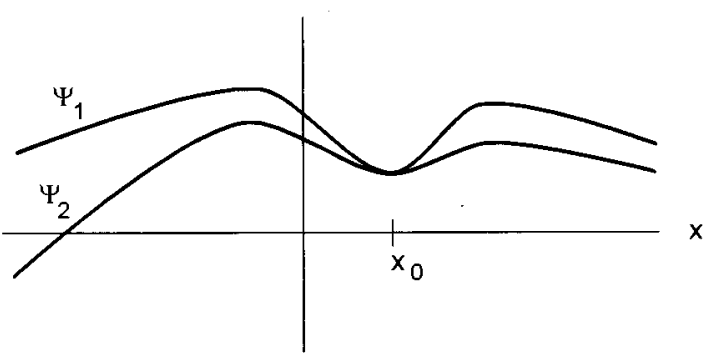

FIG. 2. The wave forms $\Psi_{1}(x, t), \Psi_{2}(x, t)$ are plotted as a function of position. The waveheight of the first exceeds that of the second everywhere except at the point $x_{0}$, where the waves kiss. Observe that the curvature of $\Psi_{1}$ (given by its second derivative at $x_{0}$ ) is larger than that of $\Psi_{2}$, leading to a larger growth rate [Eq. (3)] for $\Psi_{1}$. Hence the curves can never cross.

does not seem to possess a partial-ordering structure may in fact have a hidden ordering structure which can be revealed through a coordinate transformation. The essential point is the following: if a system can be shown to possess a partial ordering in some coordinate system, then all essential dynamical properties of partially ordered systems (to be described) will hold, regardless of reference frame. (This follows since most of these dynamical properties will be topological in nature.) In Eqs. (2), for example, upon making the coordinate transformation $u_{1}=z_{1}, u_{2}=z_{3}, u_{3}=z_{2}-\eta z_{3}$, we find that the system $\left(u_{1}, u_{2}, u_{3}\right)$ will be partially ordered provided $a>0, \lambda<0, \nu-\eta^{2} / 4<0$, and hence we have located a second parameter regime in (2) for which there can be no stable chaotic motion in the fluid [19].

(3) Reaction-diffusion systems: Subject to certain restrictions, one can show that scalar reaction-diffusion models, such as the Huxley equation [20] for signal propagation, admit a partial-ordering structure. This application of the notion of a partial ordering to continuum systems described by partial differential equations requires a slight generalization of the concepts introduced previously - we cannot directly apply the local ordering criterion described above-but is relatively straightforward. In the case of the Huxley model, the governing equation for the scalar field $\Psi$ takes the form

$$
\partial_{t} \Psi=r \Psi-\Psi^{3}+D \partial_{x x} \Psi, \quad D>0 .
$$

To prove the partial-ordering property, we show that if one wave form $\Psi_{1}(x, t)$ is initially larger than (or equal to) a second $\Psi_{2}(x, t)$ for all $x$, then it remains larger under the flow. This is most easily seen graphically. Assume that after some time the two waves become equal at a point $x_{0}$ (Fig. 2). Both waves will continue to evolve according to (3), and though their heights at the point $x_{0}$ are momentarily equal, their growth rates at $x_{0}$ will clearly differ owing to the diffusive term $D \partial_{x x} \Psi$ in (3). Since the diffusion term effectively measures the curvature of these two wave forms, it follows (Fig. 2) that the growth rate of $\Psi_{1}$ at $x_{0}$ will exceed that of $\Psi_{2}$. Hence the curves will again separate, with $\Psi_{1}$ remaining larger.

We next describe the fundamental dynamical properties shared by all physical systems which possess a partialordering structure. For completeness, we include in our discussion several results which have appeared previously in 
somewhat different contexts [6-8]. We restrict attention primarily to finite-dimensional systems described by ordinary differential equations and mappings, and will stress the geometric aspects of the partial-ordering structure.

\section{DYNAMICAL PROPERTIES OF PARTIALLY ORDERED SYSTEMS}

In analyzing the dynamical behavior of these systems, the key idea will be the notion of ordered and unordered orbits, which display markedly different behaviors. The orbit passing through an initial point $x_{0}$ is determined by the time evolution operator $\hat{\phi}_{\tau}$. An orbit is called ordered if at least two points on the orbit are comparable, otherwise it is unordered. [By a straightforward generalization, any set of points in phase space (not necessarily orbits) may be classified as being ordered or unordered, according to whether or not they contain at least two comparable points.] The partial-ordering structure will impose very different geometric constraints on these two types of orbits. We begin with the ordered orbits, and show that they have some very simple properties.

Let $x$ and $\hat{\phi}_{p}(x)$ represent two comparable points on an ordered orbit. The quantity $p$ denotes the time separation between the two, and assume $x \leq \hat{\phi}_{p}(x)$. [The case $\hat{\phi}_{p}(x) \leq x$ follows similarly.] Since by definition these points must remain ordered under time evolution, it follows that $x \leq \hat{\phi}_{p}(x) \leq \hat{\phi}_{2 p}(x) \leq \hat{\phi}_{3 p}(x) \leq \ldots$ Thus, this sequence cannot decrease with time, implying the following.

Property 1: All ordered orbits converge to a fixed point, a periodic state, or else run off to infinity.

This property states that, asymptotically, all ordered orbits are dynamically simple [21]. This statement can be refined and strengthened considerably. For instance, in the case of continuous (vector field) flows, Hirsch [6] has shown that most orbits (in a measure-theoretic sense) will converge to fixed points or infinity, not periodic orbits. We point out that this is not the case for maps, where, for example, stable 2 cycles are possible [e.g., the partially ordered mapping $x \rightarrow \tanh (x+3 y), \quad y \rightarrow \tanh (3 x+y)$ has the stable period- 2 orbit $(-0.9575,0.9575) \leftrightarrow(0.9575,-0.9575)]$.

It is useful to reconsider one of the physical examples cited previously - the case of charge-density waves. Recall that asymptotically the CDW's were found to undergo sliding motion. This thus corresponds (in Property 1) to an orbit running off to infinity. (Physically this poses no problems since the CDW variables $\phi_{i}$ represent phases.) It was also noted that all CDW's had the same time-averaged velocity, owing in part to the special discrete symmetry of Eqs. (1): $\phi_{i} \rightarrow \phi_{i}+2 \pi$ for all $i=1, \ldots, N$. This fact has a simple geometric basis. Consider any two CDW's, represented as points $\phi_{a}, \phi_{b} \in \mathbb{R}^{N}$ in phase space. By adding or subtracting enough multiples of $2 \pi$ to $\phi_{a}$, new states $\phi_{G}=\phi_{a}+2 \pi m$, $\phi_{L}=\phi_{a}-2 \pi n$ can be constructed such that the original states are both contained in the square region formed by $\phi_{G}, \phi_{L}$ (Fig. 3). Observe, in particular, that $\phi_{L} \leq \phi_{a}, \phi_{b} \leq \phi_{G}$. Under time evolution the points $\phi_{L}, \phi_{G}$ must move at the same rate due to the discrete symmetry of Eqs. (1). As they move, the points $\phi_{a}, \phi_{b}$ must remain in the square formed by $\phi_{L}, \phi_{G}$, for to leave would violate their presumed ordering with respect to $\phi_{L}, \phi_{G}$. Thus the CDW's

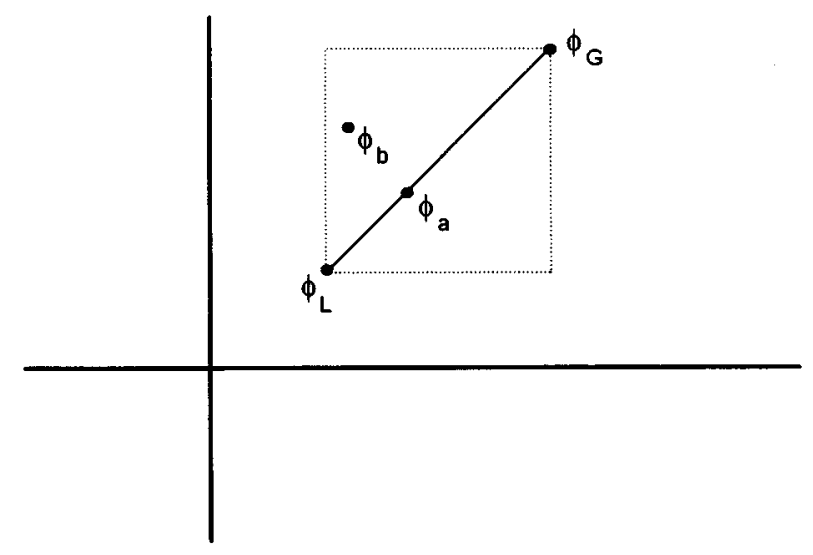

FIG. 3. Two arbitrary states $\phi_{a}, \phi_{b}$ (not necessarily ordered) can always be contained within a square region with vertex points $\phi_{G}, \phi_{L}$ defined by (respectively) adding or subtracting suitably many multiples of $2 \pi$ from one of the original points $\left(\phi_{a}\right.$ or $\left.\phi_{b}\right)$. Under the flow, this square region moves but cannot deform, with points $\phi_{a}, \phi_{b}$ always confined to this square by the partial-ordering constraint.

$\phi_{a}, \phi_{b}$ will be constrained to have the same average velocity. This is particularly important from an experimental standpoint, since it shows that the long-term behavior of a CDW will not depend on its history. Hence Middleton and Fisher's remarkable result for sliding charge-density waves is a general feature of partially ordered systems with an extra discrete symmetry. (A second example of such a system is the phase-difference equations governing the behavior of a dc-biased SQUID (superconducting quantum interference device) with an induced magnetic field. In this case, the voltage drop across the superconductor junction plays the role of the velocity.)

We next consider the unordered orbits of a partially ordered system. Since the ordered orbits have been shown to lead to simple dynamical behaviors (after transients), any complex behaviors in the system, if they are indeed possible, must reside with these unordered orbits. We first note that by Property 1 and the arguments preceding it, any orbit which doesn't converge to a fixed point, periodic state, or infinity, or any orbit which is itself periodic, is necessarily unordered. We now show that not only do such orbits exist, but that they can display arbitrarily complicated dynamics.

Property 2: Partially ordered systems can display all possible varieties of complex behaviors (e.g., chaotic dynamics, period-doubling sequences, homoclinic connections, etc.) through their unordered orbits.

We demonstrate this in a constructive fashion. Consider first the following example of a two-dimensional mapping:

$$
\begin{aligned}
& x \rightarrow r x(1+y), \\
& y \rightarrow r y(1-x),
\end{aligned}
$$

defined on the unit square $x \in[0,1], \quad y \in[-1,0]$, where for ease of exposition we assume that points mapped out of this domain are projected back to the boundary. The parameter $r$ lies in the interval $[0,4]$. The Jacobian matrix is nonnegative everywhere on the domain, and hence by the local 
ordering criterion, this map admits a partial ordering. The key observation is that the line $x+y=0$ is invariant under the dynamics, and that on this (unordered) line the mapping (4) reduces to the well-known logistic map [22], with all its concomitant behaviors (e.g., period doublings, chaos). More generally, other systems displaying arbitrarily complex behaviors can be similarly constructed by noting that, as long as all derivatives remain bounded, we have the following.

Property 3: Any $n$-dimensional dynamical system can be embedded in an $(n+1)$-dimensional partially ordered dynamical system.

This is accomplished by supplementing the original system, e.g., $x \rightarrow f(x), \quad x \equiv\left(x_{1}, x_{2}, \ldots, x_{n}\right)$, with an auxiliary equation $x_{n+1} \rightarrow K x_{n+1}$, and then transforming to new coordinates $\quad y_{i}=x_{n+1}-x_{i}, \quad(i=1, \ldots, n) ; \quad y_{n+1}=x_{1}+x_{2}$ $+\cdots+x_{n+1}$. As is easily verified, by choosing constant $K$ sufficiently large, the Jacobian matrix of the transformed system $\left(y_{1}, \ldots, y_{n+1}\right)$ can be made non-negative at all points. Geometrically, this procedure amounts to embedding an arbitrary $n$-dimensional system in an $n$-dimensional unordered surface in $\mathbb{R}^{n+1}$.

Hence partially ordered systems can describe all manner of dynamical behaviors through their unordered orbits. We will show, however, that the partial-ordering structure sets important restrictions on the geometry and stability of these unordered orbits, and describe some of the experimental ramifications of this. We begin with a geometric property peculiar to two-dimensional, partially ordered mappings.

Property 4: If a two-dimensional partially-ordered map has an unordered orbit of period three (i.e., a 3 cycle), then there exist cycles of every periodicity, as well as an uncountable set of aperiodic orbits that do not converge to any cycle.

This property is analogous to the well-known Li-Yorke theorem [13] for ordinary one-dimensional maps, showing that period three implies chaos. The proof of Property 4, which we outline here, is remarkably simple. The key step involves the construction of rectangular regions in the plane which are mapped onto one another; this allows us to use the "digraph" method of Straffin [23] as described in [24]. Let $a, b, c$ denote the three consecutive iterates making up the 3 cycle. We assume these unordered points lie as in Fig. 4(a) (other configurations can be handled similarly). Construct a rectangular region $R_{1}$ with points $a, b$ as vertices, and similarly construct $R_{2}$ from points $b, c$. Now, owing to the partial ordering structure, under one iteration of the mapping, region $R_{1}$ is mapped onto $R_{2}$. (To see this, consider, for example, the pt. $v$, which is smaller than (i.e., ordered with respect to) points $a, b$. Since points $a, b$ get mapped to $b, c$, respectively, $v$ must get mapped to a point on or outside the boundary of region $R_{2}$, in order that it still remain ordered with these points.) In a similar manner, region $R_{2}$ can be shown to be mapped onto both $R_{1}$ and $R_{2}$. We represent this schematically by a digraph [Fig. 4(b)]. Now consider any finite symbol sequence allowed by the digraph (e.g., $\left.\left\{R_{1}, R_{2}, R_{2}, R_{1}, R_{2}, R_{1}\right\}\right)$. We claim that to any such sequence there corresponds an actual orbit of the map following this path. This is easily seen by starting at the end of the symbol sequence and working backwards: In the sample sequence given above, choose any point in the ending region $R_{1}$. Then since the preceding region $R_{2}$ is mapped onto $R_{1}$, there must be some point in $R_{2}$ which is mapped to the

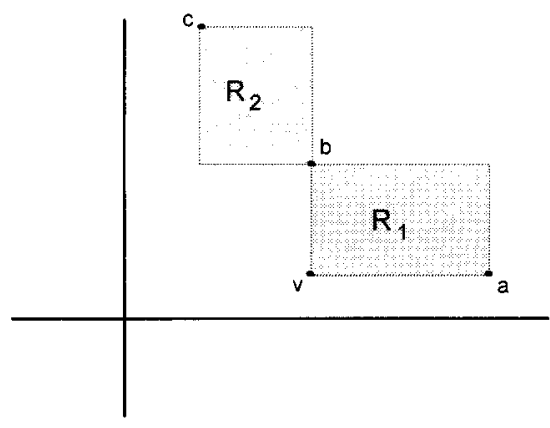

(a)

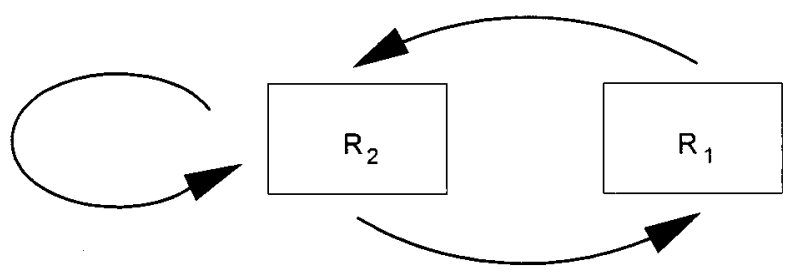

(b)

FIG. 4. (a) The point $a$ is mapped to $b, b$ to $c$, and $c$ to $a$, forming a 3 cycle. By the partial-ordering constraint, region $R_{1}$ is mapped onto region $R_{2}$, while $R_{2}$ is mapped onto both $R_{1}$ and $R_{2}$. (b) In the digraph, arrows connect regions which are mapped onto one another.

chosen point in $R_{1}$. Continuing this argument backwards through the sequence proves that the desired orbit exists. The proof for infinite sequences and periodic orbits follows similarly, using fixed point and limiting arguments. (An even stronger version of Property 4 is also possible based on the "Sarkovskii ordering" [25] of periodic orbits.)

Next, we consider one of the most significant properties of the unordered orbits in partially ordered systems from an experimental standpoint.

Property 5: All chaotic sets are nonattracting (e.g., strange attractors cannot exist).

This result implies that though chaotic unordered orbits can exist, there is no chaos for "typical" choices of initial conditions. This has immediate practical significance. Since the ordered orbits were already shown to be simple, a partial ordering in a physical system will effectively ensure that all experimentally observed behaviors will be simple (neglecting transients). For example, in the case of the three-mode fluid interaction discussed in Sec. III, we were able to predict parameter regimes for which no chaotic motions in the fluid should be observed - though we did not rule out the theoretical existence of unstable chaotic motions.

The fact that no chaotic set can be an attractor can be understood from a simple geometric argument. Suppose that a chaotic attractor $(A)$ did exist. Pick any point $\alpha$ in $A$. Now choose a second point $\beta$ which is arbitrarily close to but larger than this first point. Note that $\beta$ cannot lie in $A$ (since all points in $A$ are incomparable). Now since $\alpha$ is part of the 
attractor, there exist other points in $A$ arbitrarily close to $\alpha$. In particular, one can find a set of points in $A$ near $\alpha$, each of which is smaller than $\beta$. Now under the dynamics, $\beta$ must converge to the attractor, and so this set of points in $A$ which are less than $\beta$ must shrink (in order that they remain ordered with respect to $\beta$ ). However, since $A$ is chaotic, this set of points in $A$ will expand, leading to a contradiction. Thus chaotic attractors are not possible [26]. (This result was shown previously in $[6,27]$ by means of a different argument.) A similar argument, in conjunction with Property 1 , leads us to conjecture that almost all trajectories in the system will eventually converge to either a fixed point, periodic orbit, or infinity.

Lastly, we remark briefly on the role of partial orderings on the pattern-forming process in physical systems. It is well known that the formation of patterns and other coherent structures (in fluids, chemical systems, lasers, etc.) is intimately connected with instabilities in the system (see, e.g., [28]). The two most common forms of instabilities are steady state bifurcations and oscillatory (Hopf) bifurcations, each giving rise to a different class of patterns. Convection in a fluid illustrates both types: if a layer of fluid is heated from below, then for sufficiently low heating rates there is no motion in the fluid and heat is simply conducted upwards. Above a certain critical heating rate, however, the conduction state becomes unstable, and a transition occurs: in the steady state case, a pattern of stationary Rayleigh-Bénard rolls emerges, with fluid upswelling in some regions and downswelling in others, while in the oscillatory case, rolls also appear, but now their sense of rotation (clockwise or counterclockwise) can oscillate in time, or the whole pattern can start drifting (leftward or rightward). The former is associated with a real eigenvalue of the linearized equations of motion becoming positive, while the latter occurs when the real part of a complex eigenvalue becomes positive. We observe, however, that in a partially ordered system, the eigenvalues will be restricted by the non-negativity constraint on the Jacobian matrix: a well-known result from matrix theory (see [29]) says that a matrix with non-negative elements will have a real, non-negative eigenvalue which exceeds all oth- ers in modulus. From this, it immediately follows that the first instability to appear in a partially ordered system will (typically) be of the steady state bifurcation variety-the partial ordering prevents the oscillatory bifurcation from setting in first.

\section{DISCUSSION}

Though not often recognized, partial-ordering structures lie hidden in many physical systems of significant interest, and have important implications in terms of the possible dynamical behaviors of the system. Once a partial-ordering structure has been identified, it immediately follows that most observable behaviors in the system will be simple, even though complicated dynamical behavior is theoretically allowed. In our discussion we have tried to emphasize the geometric aspects of the theory, which is perhaps the most natural as well as most versatile approach. This area remains largely unexplored, however. Particularly interesting are questions concerning orderings in continuum models such as fluids and other extended media. Defining a partial-ordering relation for such continuum systems is a simple matter, and many of the results described here for finite-dimensional systems carry over to the continuum case [8]. However, the analysis becomes much more delicate, and several new features can emerge: e.g., an ordered orbit in such systems can converge to a propagating wave front, in addition to the limiting states noted in Property 1. Lastly, we remark that what is perhaps most surprising is how simple it is to discover partial orderings in many well-known physical and mathematical systems: for example, subject to certain restrictions, Burger's equation [30], Fisher's equation [31], and the SinhGordon equation [32] all contain partial-ordering structures.

\section{ACKNOWLEDGMENTS}

We gratefully acknowledge useful discussions with Morris Hirsch, Kevin McCardle, and Kurt Wiesenfeld. This work was supported in part by the Office of Naval Research, Grant No. N00014-J-1257.
[1] A. Tarski, Pac. J. Math. 5, 285 (1955).

[2] S. Lippman, J. Mamer, and K. McCardle, J. Econ. Theory 41, 288 (1987).

[3] D. M. Topkis, SIAM (Soc. Ind. Appl. Math) J. Control Optim. 17, 773 (1979).

[4] P. Milgrom and J. Roberts, Econometrica 58, 1255 (1990).

[5] X. Vives, J. Math. Econ. 19, 305 (1990).

[6] M. Hirsch, SIAM (Soc. Ind. Appl. Math.) J. Math. Anal. 13, 167 (1982).

[7] M. Hirsch, SIAM (Soc. Ind. Appl. Math.) J. Math. Anal. 16, 423 (1985).

[8] M. Hirsch, J. Reine Angew. Math. 383, 1 (1988).

[9] H. L. Smith, Monotone Dynamical Systems: An Introduction to the Theory of Competitive and Cooperative Systems, Vol. 41 of Mathematical Surveys and Monographs (American Mathematical Society, Providence, 1995).
[10] E. Friedman and A. S. Landsberg, Oper. Res. Lett. 14, 221 (1993).

[11] E. Friedman and A. S. Landsberg, Oper. Res. Lett. 18, 185 (1996).

[12] E. Friedman (unpublished).

[13] T. Y. Li and J. A. Yorke, Am. Math. Mon. 82, 985 (1975).

[14] E. Kamke, Acta Math. 58, 57 (1932).

[15] A. A. Middleton, Phys. Rev. Lett. 68, 670 (1992).

[16] A. A. Middleton and D. S. Fisher, Phys. Rev. B 47, 3530 (1993).

[17] A. Arneodo, P. H. Coullet, and E. A. Spiegel, Geophys. Astrophys. Fluid Dyn. 31, 1 (1985).

[18] P. Glendinning and C. Sparrow, J. Stat. Phys. A 35, 645 (1984).

[19] A. S. Landsberg, Ph.D. thesis, University of California at Berkeley, 1994. 
[20] P. Fife, Mathematical Aspects of Reacting and Diffusing Systems (Springer-Verlag, New York, 1979).

[21] An interesting twist occurs if instead of Cartesian phase space we consider toroidal phase spaces. Strictly speaking, partial orderings cannot be defined on such spaces, but this can be circumvented by first "lifting"' to $\mathbb{R}^{n}$, and then subsequently projecting back down. Owing to this projection, however, our stated results must be reinterpreted accordingly. This is a simple matter, yet nonetheless has interesting consequences. One can show, for instance, that Property 1 no longer always holds, and chaotic ordered orbits become possible.

[22] R. M. May, Nature 261, 459 (1976).

[23] P. D. Straffin, Math. Mag. 51, 99 (1978).

[24] E. A. Jackson, Perspective in Nonlinear Dynamics: Volume 1 (Cambridge University Press, New York, 1989).

[25] A. N. Sarkovskii, UKr. Mat. Zh. 16, 61 (1964).
[26] In fact, even stronger statements can be made. For instance, all chaotic sets will be repelling, with the rate of repulsion being at least as large as the rate of expansion (due to chaos) within the set [Friedman and Landsberg (unpublished)]. Also, as shown by Hirsch [6], the fractal (Hausdorff) dimension of such sets in $n$-dimensional phase space is at most $n-1$.

[27] M. Hirsch, Bull. Am. Math. Soc. 11, 1 (1984).

[28] M. C. Cross and P. C. Hohenberg, Rev. Mod. Phys. 65, 851 (1993).

[29] F. R. Gantmacher, Applications of the Theory of Matrices (Interscience, New York, 1959).

[30] E. R. Benton and G. W. Platzman, Quart. Appl. Math. 30, 195 (1972).

[31] P. Kaliappan, Physica D 11, 368 (1984).

[32] A. Grauel, Physica A 132, 557 (1985). 